\title{
Basic Reproduction Number of Chikungunya Virus Transmitted by Aedes Mosquitoes
}

\author{
Najmul Haider, Francesco Vairo, Giuseppe Ippolito, Alimuddin Zumla, Richard A. Kock
}

We estimated the weighted mean basic reproduction number $\left(R_{0}\right)$ of chikungunya virus based on outbreak size. $\mathrm{R}_{0}$ was 3.4 (95\% Cl 2.4-4.2) and varied for 2 primary chikungunya mosquito vectors: 4.1 (95\% Cl 1.5-6.6) for $\mathrm{Ae}$ des aegypti and $2.8(95 \% \mathrm{Cl} 1.8-3.8)$ for Ae. albopictus.

$\mathrm{T}$ he basic reproduction number $\left(\mathrm{R}_{0}\right)$ of an infection is the mean number of secondary cases a single infectious person causes in a completely susceptible population. The magnitude of $R_{0}$ is used to measure the risk and spread of an epidemic or pandemic. To control an outbreak, the $R_{0}$ should be reduced to $\leq 1$ through interventions, such as vaccination. Because little information is available at the beginning of an epidemic, the estimated $R_{0}$ commonly is used to assess public health preparedness needs, the impact of the possible epidemic, and success of the control measures. Information on $\mathrm{R}_{0}$ often is lacking for emerging diseases like chikungunya, a mosquito-borne viral disease of humans and nonhuman primates.

Chikungunya virus (CHIKV) is a member of the Alphavirus genus (family Togaviridae) transmitted by Aedes mosquitoes, primarily Ae. aegypti and Ae. albopictus. Ae. aegypti mosquitoes are aggressive human biters and the main vectors for CHIKV outbreaks in Asia, where epidemics occur primarily in urban settings (1). Ae. albopictus mosquitoes, on the other hand, feed from several mammals besides humans and are responsible for $\mathrm{CHIKV}$ outbreaks in rural and urban areas in Africa (1).

CHIKV outbreaks were reported from $>100$ countries worldwide during 2014-2019 (2). Epidemiologic understanding of CHIKV changed after outbreaks on the island of La Réunion in the Indian Ocean

Author affiliations: The Royal Veterinary College, University of London, London, UK (N. Haider, R.A. Kock); National Institute for Infectious Diseases Lazzaro Spallanzani, Rome, Italy (F. Vairo, G. Ippolito); University College London, London (A. Zumla)

DOI: https://doi.org/10.3201/eid2610.190957 during 2005-2006, when Ae. albopictus mosquitoes were identified as the outbreak vector $(1,3)$. The global expansion of CHIKV partially is attributed to viral adaptation to this new mosquito vector, which facilitated a mutation in the coding for the envelop protein $1 \mathrm{~A} 226 \mathrm{~V}$ (E1-A226V) gene of CHIKV, increasing the competence of Ae. albopictus mosquitoes to transmit the virus from mosquitoes to humans (1-3).

In humans, CHIV infection is characterized by sudden onset of intense polyarthralgia, high fever, and skin rash. CHIKV causes debilitating joint pain that can limit daily activities and last a few months to several years (2); progression to the chronic stage (>3 months) occurs in $4.1 \%-78.6 \%$ of cases (4). To estimate $\mathrm{R}_{0}$ of CHIKV outbreaks, we analyzed empirical data on $R_{0}$ available from open sources.

\section{The Study}

We used the search terms "Basic reproduction number" or "R" AND "chikungunya" to identify published articles from Google Scholar and PubMed. We identified 11 articles describing estimated $\mathrm{R}_{0}$ of $\mathrm{CHIKV}$ from outbreak data during 2000-2019. We found 5 articles on outbreaks in Africa, all on La Réunion (3,5-8); 1 on an outbreak in Cambodia (1); 2 on outbreaks in Italy $(9,10)$; and 3 on outbreaks in the Americas $(11,12$; N. Báez-Hernández et al. unpub. data, https:/ / www. biorxiv.org/content/10.1101/122556v1).

The authors estimated $R_{0}$ by using mathematical (compartmental) models fitted with respective outbreak data (1,3,5-12). We considered the estimated values comparable and extracted the $R_{0}$ from each. We then estimated the weighted mean $\mathrm{R}_{0}$ of $\mathrm{CHIKV}$ based on outbreak size, such as number of reported cases included in the estimation of $R_{0}$ in the original article, and further estimated the mean $\mathrm{R}_{0}$ for different mosquito vectors and E1-A226V gene mutations.

The largest CHIKV outbreak occurred on La Réunion and affected 266,000 of the 785,000 inhabitants (3). Several models with differing levels of data 
estimated the $\mathrm{R}_{0}$ of the La Réunion outbreaks between 0.89 and $4.1(3,5-7)$. The $R_{0}$ also was estimated from CHIKV outbreaks in Italy in 2007 (10) and 2017 (9), Cambodia in 2012 (1), Venezuela in 2014 (11), Colombia in 2015 (12), and Mexico in 2015 (N. Báez-Hernández et al. unpub. data, https://www.biorxiv.org/ content/10.1101/122556v1) (Table).

We estimated the weighted mean $\mathrm{R}_{0}$ of CHIKV to be 3.4 (95\% CI 2.4-4.2). We analyzed the data and estimated the $\mathrm{R}_{0}$ for Ae. aegypti and Ae. albopictus mosquitoes separately for outbreaks in which the $R_{0}$ of CHIKV was described for each species. We estimated the $\mathrm{R}_{0}$ to be 4.1 (95\% CI 1.5-6.6) for Ae. aegypti mosquitoes and 2.8 (95\% CI 1.8-3.8) for Ae. albopictus mosquitoes. Although the difference is not statistically significant $(p=0.12)$, we expected a lower $R_{0}$ for outbreaks involving Ae. albopictus mosquitoes because this species also feeds on animals, which might have reduced the attack rate on humans and transmission across the population. However, outbreaks associated with Ae. albopictus mosquitoes can be prolonged and the outbreak response can have economic consequences. We estimated the $\mathrm{R}_{0}$ to be 3.5 (95\% CI 1.9-4.9) during outbreaks involving the E1-A226V mutation, which is higher than $R_{0}$ of 2.1 from the 2017 outbreak in Italy that did not have the gene mutation.

CHIKV infections among humans can have severe health consequences, despite the low case fatality rate. CHIKV infection has 3 stages: acute, postacute, and chronic. The acute phase usually lasts for 1-3 weeks and is characterized by fever, intense myalgia, arthralgia, and symmetric joint pain in both legs that can limit even the simplest daily activities. The postacute stage usually lasts 1-3 months after the acute phase and is characterized by persistent inflammatory arthralgia, arthritis, tenosynovitis, and bursitis. The chronic stage starts after 3 months and can last for months to years after acute infection (2).

In a study in Brazil, $\geq 68 \%$ of persons with CHIKV remained chronically infected for up to 1 year (13). On
Réunion Island, a small group of patients had clinical signs for 6 years. Although the reason for persistence is unclear, it might be strain related and associated with the E1-A226V mutation. Therefore, despite being less severe and causing fewer deaths than other mosquitoborne diseases, CHIKV can have lingering physical and psychological consequences for those affected. Infected persons also can experience economic consequences because they might not be able to work for several weeks or more.

$\mathrm{R}_{0}$ does not remain constant. For arboviruses, $\mathrm{R}_{0}$ can vary based on the density of hosts and vectors; mosquito species, survival, and biting rate; and vector competence and capacity, all of which can depend on environmental and microclimatic factors. Further, the vector competence of Ae. aegypti mosquitoes for CHIKV might be different from that for Ae. albopictus mosquitoes, which could influence outbreak dynamics. For example, 1 study reported the transmission efficiency of Ae. albopictus mosquitoes as $97 \%$ and of Ae. aegypti mosquitoes as $83 \%$ (14).

The outbreaks included in our study occurred in tropical and subtropical countries and in the more temperate climate of Italy. We did not consider climatic conditions during reported outbreaks, which might play a role in determining the size and $R_{0}$ of CHIKV outbreaks. We also did not consider the variation of data quality in published articles, except for the outbreak size, which might affect estimated $\mathrm{R}_{0}$. However, defining adjustments for data quality would have been difficult and might have introduced unwanted bias.

\section{Conclusions}

We found the overall mean $\mathrm{R}_{0}$ for CHIKV was 3.4 (95\% CI 2.4-4.2). Our estimated $R_{0}$ of 4.1 (95\% CI 1.56.6) for Ae. aegypti mosquitoes suggests CHIKV could spread rapidly and cause high disease incidence in urban areas, where this species thrives. Our estimated CHIKV R $\mathrm{R}_{0}$ for Ae. albopictus mosquitoes of 2.8 (95\%

\begin{tabular}{|c|c|c|c|c|c|c|c|}
\hline Year & Country or region & Continent & $\mathrm{R}_{0}$ range $(95 \% \mathrm{Cl})$ & $\begin{array}{c}\text { Mosquito } \\
\text { species }\end{array}$ & Lineage & $\begin{array}{l}\text { E1-A226V } \\
\text { mutation* }\end{array}$ & Reference \\
\hline 2006 & La Réunion & Africa & 4.1 & Ae. albopictus & Indian Ocean & $\mathrm{Y}$ & (3) \\
\hline 2006 & La Réunion & Africa & $0.9-2.3$ & Ae. albopictus & Indian Ocean & $Y$ & (7) \\
\hline 2006 & La Réunion & Africa & $1.5-1.8$ & Ae. albopictus & Indian Ocean & Y & (5) \\
\hline 2006 & La Réunion & Africa & 3.4 & Ae. albopictus & Indian Ocean & $\mathrm{Y}$ & (6) \\
\hline 2006 & La Réunion & Africa & $3.7(2-11)$ & Ae. albopictus & Indian Ocean & Y & (8) \\
\hline 2007 & Italy & Europe & $3.3(1.8-6.0)$ & Ae. albopictus & Indian Ocean & Mixed & $(10)$ \\
\hline 2012 & Cambodia & Asia & $6.5(6.2-6.8)$ & Ae. aegypti & Asian & Y & $(1)$ \\
\hline 2014 & Italy & Europe & $2.1(1.5-2.6)$ & Ae. albopictus & Indian Ocean & $\mathrm{N}$ & (9) \\
\hline 2014 & Venezuela & South America & 3.7 & Ae. aegypti & Asian & $\mathrm{N}$ & (11) \\
\hline 2015 & Mexico & North America & 3.44 & Ae. aegypti & Asian & $\mathrm{N}$ & $\dagger$ \\
\hline 2014 & Colombia & South America & $1-9$ & Ae. aegypti & Asian & $\mathrm{N}$ & (12) \\
\hline
\end{tabular}

*Envelope $1 \mathrm{~A} 226 \mathrm{~V}$ gene.

†N. Báez-Hernández et al., unpub data, https://www.biorxiv.org/content/10.1101/122556v1. 
CI 1.5-6.6) was lower than for Ae. aegypti mosquitoes. In rural areas, where Ae. albopictus mosquitoes are more prevalent, sylvatic cycles, maintenance of biodiversity including natural mosquito populations, and presence of hosts other than humans might reduce the effects of an outbreak. Early interventions targeting Aedes mosquitoes will be vital to controlling CHIKV outbreaks.

All authors are part of PANDORA-ID-NET Consortium funded by the European and Developing Countries Clinical Trials Partnership (EDCTP2) program (EDCTP grant no. RIA2016E-1609), which is supported under Horizon 2020, the European Union's Framework Programme for Research and Innovation. The members were part of an international outbreak response on chikungunya virus outbreaks in Republic of Congo.

G.I. and F.V. acknowledge support for research on emerging infections from the Italian Ministry of Health, through grants to Ricerca Corrente linea 1 to National Institute for Infectious Diseases, Lazzaro Spallanzani, IRCCS, Rome. G.I., F.V., and A.Z. are members of the International Public Health Crisis Group that performs independent analysis and research activities on epidemic and endemic events naturally occurring or related to the deliberate release of pathogens, and provides strategic, organizational, educational, logistic support, and advice for preparedness and response. F.V. and G.I. are professors at Saint Camillus International University of Health Sciences, Rome, Italy.

\section{About the Author}

Dr. Haider is a veterinarian, epidemiologist, and public health researcher based in the Royal Veterinary College, London, UK. His research interests focus on emerging infectious diseases, including chikungunya and Lassa fever virus, and interrupting the chain of transmission.

\section{References}

1. Robinson M, Conan A, Duong V, Ly S, Ngan C, Buchy P, et al. A model for a chikungunya outbreak in a rural Cambodian setting: implications for disease control in uninfected areas. PLoS Negl Trop Dis. 2014;8:e3120. https:/ / doi.org/10.1371/journal.pntd.0003120

2. Vairo F, Haider N, Kock R, Ntoumi F, Ippolito G, Zumla A. Chikungunya: epidemiology, pathogenesis, clinical features, management, and prevention. Infect Dis Clin North Am. 2019;33:1003-25. https://doi.org/10.1016/j.idc.2019.08.006
3. Yakob L, Clements ACA. A mathematical model of chikungunya dynamics and control: the major epidemic on Réunion Island. PLoS One. 2013;8:e57448.

https://doi.org/10.1371/journal.pone.0057448

4. Rodríguez-Morales AJ, Cardona-Ospina JA, Fernanda Urbano-Garzón S, Sebastian Hurtado-Zapata J. Prevalence of post-chikungunya infection chronic inflammatory arthritis: a systematic review and meta-analysis. Arthritis Care Res (Hoboken). 2016;68:1849-58. https://doi.org/10.1002/acr.22900

5. Dumont $Y$, Chiroleu F. Vector control for the chikungunya disease. Math Biosci Eng. 2010;7:313-45. https:/ / doi.org/ 10.3934/mbe.2010.7.313

6. Bacaër N. Approximation of the basic reproduction number $\mathrm{R}_{0}$ for vector-borne diseases with a periodic vector population. Bull Math Biol. 2007;69:1067-91. https://doi.org/10.1007/s11538-006-9166-9

7. Dumont Y, Chiroleu F, Domerg C. On a temporal model for the chikungunya disease: modeling, theory and numerics. Math Biosci. 2008;213:80-91. https:/ / doi.org/ 10.1016/j.mbs.2008.02.008

8. Boëlle P-Y, Thomas G, Vergu E, Renault P, Valleron A-J, Flahault A. Investigating transmission in a two-wave epidemic of chikungunya fever, Réunion Island. Vector-Borne Zoonotic Dis. 2008;8:207-17. https:// doi.org/10.1089/vbz.2006.0620

9. Manica M, Guzzetta G, Poletti P, Filipponi F, Solimini A, Caputo B, et al. Transmission dynamics of the ongoing chikungunya outbreak in Central Italy: from coastal areas to the metropolitan city of Rome, summer 2017. Euro Surveill. 2017;22:17-00685. https:// doi.org/10.2807/15607917.ES.2017.22.44.17-00685

10. Poletti P, Messeri G, Ajelli M, Vallorani R, Rizzo C, Merler S. Transmission potential of chikungunya virus and control measures: the case of Italy. PLoS One. 2011;6:e18860. https://doi.org/10.1371/journal.pone.0018860

11. Lizarazo E, Vincenti-Gonzalez M, Grillet ME, Bethencourt S, Diaz O, Ojeda N, et al. Spatial dynamics of chikungunya virus, Venezuela, 2014. Emerg Infect Dis. 2019;25:672-80. https://doi.org/10.3201/eid2504.172121

12. Peña-García VH, Christofferson RC. Correlation of the basic reproduction number $\left(\mathrm{R}_{0}\right)$ and eco-environmental variables in Colombian municipalities with chikungunya outbreaks during 2014-2016. PLoS Negl Trop Dis. 2019;13:e0007878. PubMed https:/ / doi.org/10.1371/journal.pntd.0007878

13. Dias JP, Costa MCN, Campos GS, Paixão ES, Natividade MS, Barreto FR, et al. Seroprevalence of chikungunya virus after its emergence in Brazil. Emerg Infect Dis. 2018;24:617-24. PubMed https:/ / doi.org/10.3201/eid2404.171370

14. Vega-Rúa A, Zouache K, Girod R, Failloux A-B, Lourençode-Oliveira R. High level of vector competence of Aedes aegypti and Aedes albopictus from ten American countries as a crucial factor in the spread of chikungunya virus. J Virol. 2014;88:6294-306. https:// doi.org/10.1128/JVI.00370-14

Address for correspondence: Najmul Haider, Centre for Emerging, Endemic and Exotic Diseases, Room no. CEE 001, Royal Veterinary College, University of London, Hawkshead Ln, North Mymms, Hertfordshire AL9 7TA, UK; email: nhaider@rvc.ac.uk 